\title{
Reactive oxygen species responsive nanoplatforms as smart drug delivery systems for gastrointestinal tract targeting
}

\begin{abstract}
Serena Bertoni $^{1,2}$ | Ariella Machness $^{1,3}$ | Mattia Tiboni ${ }^{1,4}$ | Raquel Bártolo ${ }^{1}$ |
Hélder A. Santos ${ }^{1,5}$

${ }^{1}$ Drug Research Program, Division of Pharmaceutical Chemistry and Technology, Faculty of Pharmacy, University of Helsinki, Helsinki, Finland

${ }^{2}$ Department of Pharmacy and Biotechnology, University of Bologna, Bologna, Italy

${ }^{3}$ Department of Materials Science and Engineering, University of California Los Angeles, Los Angeles, California, USA

${ }^{4}$ Department of Biomolecular Sciences, University of Urbino Carlo Bo, Urbino, Italy

${ }^{5}$ Helsinki Institute of Life Science (HiLIFE),

University of Helsinki, Helsinki, Finland

\section{Correspondence}

Hélder A. Santos, Drug Research Program, Division of Pharmaceutical Chemistry and Technology, Faculty of Pharmacy, University of Helsinki, Helsinki 00014, Finland.

Abstract

The pharmacological therapy for gastrointestinal (GI) diseases, such as inflammatory bowel diseases, continues to present challenges in targeting efficacy. The need for maximal local drug exposure at the inflamed regions of the $\mathrm{Gl}$ tract has led research to focus on a disease-targeted drug delivery approach. Smart nanomaterials responsive to the reactive oxygen species (ROS) concentrated in the inflamed areas, can be formulated into nanoplatforms to selectively release the active compounds, avoiding unspecific drug delivery to healthy tissues and limiting systemic absorption. Recent developments of ROS-responsive nanoplatforms include combination with other materials to obtain multi-responsive systems and modifications/derivatization to increase the interactions with biological tissues, cell uptake and targeting. This review describes the applications of ROS-responsive nanosystems for on-demand drug delivery to the $\mathrm{Gl}$ tract.
\end{abstract}

Email: helder.santos@helsinki.fi

Funding information

HiLIFE Research Funds, Grant/Award Number: NA; Italian Ministry of Education, University and Research for the "Bando Leonardo da Vinci

- Azione 2" travel grant; Sigrid Juséliuksen Säätiö; Suomen Akatemia, Grant/Award Number: 319330

\section{KEYWORDS}

gastrointestinal tract drug delivery, irritable bowel disease, oxidation-responsive materials, stimuli-responsive nanocarriers

\section{1 | INTRODUCTION}

Localized gastrointestinal (GI) therapy has important applications in a wide array of diseases affecting the upper part (e.g., gastric ulcers) and the middle-to-lower regions of the intestine, such as inflammatory bowel disease (IBD), irritable bowel syndrome (IBS), colon cancer and infections. In these cases, the conventional oral therapy is discouraged, because of the adverse effects associated with systemic exposure. The available platforms for local GI therapy aim at controlling the drug release by exploiting environmental features, such as $\mathrm{pH}$ (e.g., pH-dependent or enteric-coated formulations), transit time (e.g., time-delayed systems), presence of mucus (e.g., bioadhesive formulations) or enzymes produced by colonic bacteria (e.g., prodrugs

Serena Bertoni, Ariella Machness, and Mattia Tiboni contributed equally to this work. and enzymes-activated systems). ${ }^{[1,2]}$ Although these strategies provide advantages over traditional systemic dosage forms in terms of reduced toxicity and unwanted side effects, these medications still suffer from lack of therapeutic efficacy, difficulty of drug targeting, insufficient selectivity, low drug retention at the inflamed areas and high variability in local drug concentrations. Such challenges are present because these targeting strategies rely on physiological parameters, which are highly variable between patients.

To overcome challenges associated with pathophysiological variations in patients, research is recently moving from strategies based on site-specific targeting to a disease-specific targeting approach, where the objective is to achieve a selective drug release only in the diseased regions. To this purpose, the environmental features of the tissues affected by the disease can be used as a trigger to activate drug release. Specifically, the abnormally high levels of reactive oxygen 
species (ROS), which characterize the microenvironment of gastric and intestinal inflamed regions, can be exploited to differentiate and target diseased tissues from healthy ones. ROS-responsive (also defined as redox- or oxidation-responsive) drug delivery systems represent a type of stimuli-responsive systems, based on materials which can change their physicochemical properties in response to the specific environment. $^{[3]}$

This new approach of developing nanosystems based on ROSresponsive materials is gaining attention for its potential to enhance the drug availability at the site of action, allowing not only to reduce the dose to be administered, but minimize the undesired side effects and improve the therapeutic efficacy. As for the most of nanoformulations developed, a basic characterization of the nanosystem should always report a minimum quantity of information including the initial material characterization with the synthesis of new materials, definition of size, shape, morphology and zeta-potential, drug loading and release. ${ }^{[4]}$

This review will focus on ROS-responsive nanosystems for GI drug targeting. After giving a background on advantages and drawbacks in GI drug delivery, we provide an overview of the ROS-responsive nanoplatforms explored so far and describe their composition, design principles and potential for local GI therapy.

\section{2 | DRUG DELIVERY TO THE GIT}

The digestive tract consists of a hollow muscular tube composed of the upper (oral cavity, esophagus, stomach and duodenum) and lower (small intestine, cecum, colon, rectum, and anus) parts, and their functions include nutrient digestion and absorption, waste excretion, and immunity. The intestinal mucosa is a complex tissue representing a selectively permeable barrier between the external environments and the internal milieu. ${ }^{[5]}$ The epithelium is a single-cell layer composed of different cell subtypes, mainly enterocytes (absorptive cells), but also goblet cells, Paneth cells, enteroendocrine cells, M-cells, cup cells and Tuft cells. ${ }^{[6]}$ The presence of tight junctions (TJs), which strongly seal the spaces between epithelial cells and ensures the intestinal barrier's function. Moreover, the epithelium is covered by a protective mucus layer, a viscous fluid secreted by goblet cells mainly composed of mucin glycoproteins. ${ }^{[7]}$ Despite these natural barriers, the GI tract represents a convenient way of access for therapeutic molecules. Among the different administration routes, the oral pathway is considered the most common and preferred route, and can be used to achieve either a systemic effect by absorption of the active compound or a local therapy by delivering the drug to the lumen and/or the membrane of the $\mathrm{Gl}$ tract. $^{[8]}$ The latter effect is desirable in $\mathrm{Gl}$ diseases, where the objective is to increase the topical drug exposure and limit systemic absorption. To maximize the local therapeutic response and limit the systemic toxicity, the formulation should meet two main requirements:

i. travel through the $\mathrm{Gl}$ tract and maintain drug integrity until it reaches the inflamed areas; and ii. make the drug available at the inflamed sites at concentrations sufficient to provide the therapeutic response, while not permeate through the intestinal mucosa.

There are, however, several key challenges that must be overcome to achieve a successful targeted drug delivery to specific intestinal regions. After ingestion during its transit through the $\mathrm{Gl}$ tract, the drug encounters various compartments, as shown in Figure 1.

The first major challenge is represented by the stomach, where the $\mathrm{pH}$ is generally low and can vary between 1 and 3.5, depending on the fasted/fed states. ${ }^{[9]}$ Moreover, the gastric enzymes, mainly pepsin, constitute another possible cause for drug instability. Later, the passage from the stomach to the small intestine causes a shift of the $\mathrm{pH}$ to weakly acidic/neutral values (5.0 and 7.0). ${ }^{[10]}$ The intestine is a dynamic environment characterized by absorptive structures (e.g., microvilli) and an intense motility provided by coordinated contractions (peristalsis). Both features can contribute to limiting the accumulation of drug at local intestinal sites. Moreover, drug stability can be affected by intestinal fluid components, such as bile salts and digestive enzymes (hydrolases, lipases, peptidases, and amylases). When the drug is released in the intestinal lumen, it is generally difficult to completely avoid drug absorption, especially when the active compound is a highly permeable molecule. For example, budesonide (BDS), a synthetic steroid indicated for the treatment of intestinal inflammation, has limited systemic exposure due to its marked hepatic metabolism (about 90\%). Despite this, the systemic availability of BDS after a single oral administration of capsules was on the order of $10 \%$ to $14 \% .{ }^{[11]}$ Unlike the small intestine, the large intestine is not the primary site of nutrient absorption, but plays a major role in water absorption and metabolism of undigested material through the intestinal microbiome. The gut microbiota consists of $10^{13}-10^{14}$ microorganisms, which provide active metabolic activity, playing a crucial role in intestinal barrier function, energy balance, and immune response. ${ }^{[12]}$ Within the large intestine the $\mathrm{pH}$ drops to slightly acidic (6.0-6.5) and the motility is reduced.

A distinction in therapeutic delivery should be made based on the specific target site. While for some active pharmaceutical ingredients delivery to the lumen is sufficient (e.g., antibiotics), in the case of antiinflammatory drugs (e.g., 5-ASA, steroids) and macromolecules, the target is more likely to be within the epithelium or submucosa. ${ }^{[13]}$ While in the former case a delayed- or $\mathrm{pH}$-dependent formulation is typically sufficient to reach the inflamed site, the latter case is more challenging, because the drug needs to overcome the mucus barrier to reach the epithelial cell membrane In instances where binding to an intended receptor is required, the drug should enter the intestinal cells through the apical side (i.e., intracellular drug release). Simultaneously, the permeability across the basolateral membrane should be limited. ${ }^{[13]}$

Moreover, a diseased state could profoundly affect GI physiology, including the transit time, mobility, composition and $\mathrm{pH}$ of the $\mathrm{GI}$ fluids, and gut microbiota (Figure 1). These changes, together with the high variability in the location and extent of intestinal lesions, ${ }^{[14]}$ represent important challenges in realizing successful therapeutic 


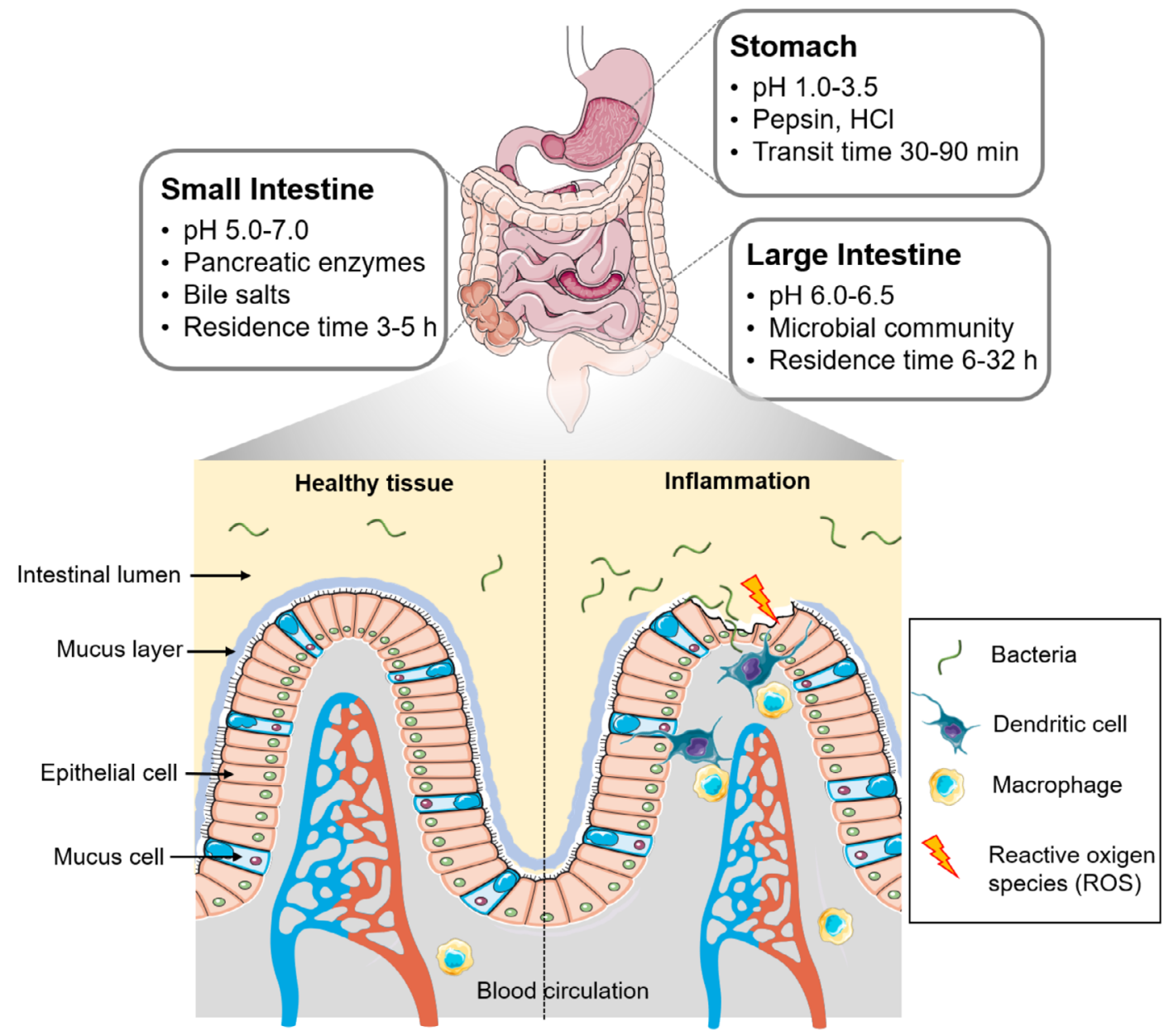

FIGURE 1 Different Gl environment encountered by oral formulations and representation of the intestinal mucosa in either healthy or inflammatory conditions. Figure generated with Servier Medical Art (SMArt)

platforms. Specifically, common alterations in Gl diseases are increased mobility, diarrhea and changes in microbiome composition (dysbiosis). ${ }^{[15]}$ For instance, it has been reported that IBD patients had significantly higher colon transit time, about 52 hours compared to healthy subjects (about 24 hours), ${ }^{[16]}$ leading to decreased contact time of the dosage form with the intestinal mucosa and thus the local drug bioavailability. The alteration in mucus layer composition and thickness, TJs disruption, epithelial lesions and ulcers can determine a partial loss of barrier integrity with increased permeability. Moreover, at the sites of active inflammation, the infiltration of immune cells (e.g., neutrophils, macrophages, and dendritic cells) is accompanied by an important release of inflammatory mediators, such as cytokines and ROS. Specifically, the intestinal mucosa of patients with IBD, ${ }^{[17]}$ cancer, ${ }^{[18]}$ as well as other $\mathrm{Gl}$ diseases showed oxidative stress with increased ROS levels. Biopsies taken from patients suffering from ulcerative colitis have a 10- to 100 -fold increase in mucosal ROS concentrations, which are confined to sites of disease and correlate with disease progression. Activated phagocytes are typically the main producers of high concentrations of ROS localized at intestinal inflammation sites. ${ }^{[15]}$

\section{3 | ROS-RESPONSIVE MATERIALS}

As previously discussed, the GI tract is an important source of ROS. ROS include radicals [superoxide $\left(\mathrm{O}_{2}{ }^{\circ}\right)$ and hydroxyl $\left({ }^{\circ} \mathrm{OH}\right)$ ] and nonradicals, such as singlet oxygen $\left({ }^{1} \mathrm{O}_{2}\right)$ and hydrogen peroxide $\left(\mathrm{H}_{2} \mathrm{O}_{2}\right) .{ }^{[17]}$ They participate in a variety of physiological processes, including cellular signaling, immune response and metabolism. However, under pathological conditions ROS may lead to cellular damage, initiation and progression of inflammation. ${ }^{[19]}$ ROS-responsive or redox-responsive materials are a new generation of engineered materials, generally of polymeric nature, that changes their chemical structure in response to one or more types of ROS. The reader interested in details on the chemical structures and mechanisms of oxidationresponsiveness of the various types of ROS-responsive materials is referred to recent reviews. ${ }^{[19-21]}$

In general, ROS-responsive polymers can be divided in two main categories: those responding to the oxidative environment with a solubility switch, and those containing ROS-cleavable bonds (Figure 2). In the first case, oxidative conditions cause a hydrophobic-hydrophilic transition, which allows the polymer solubilization in aqueous 


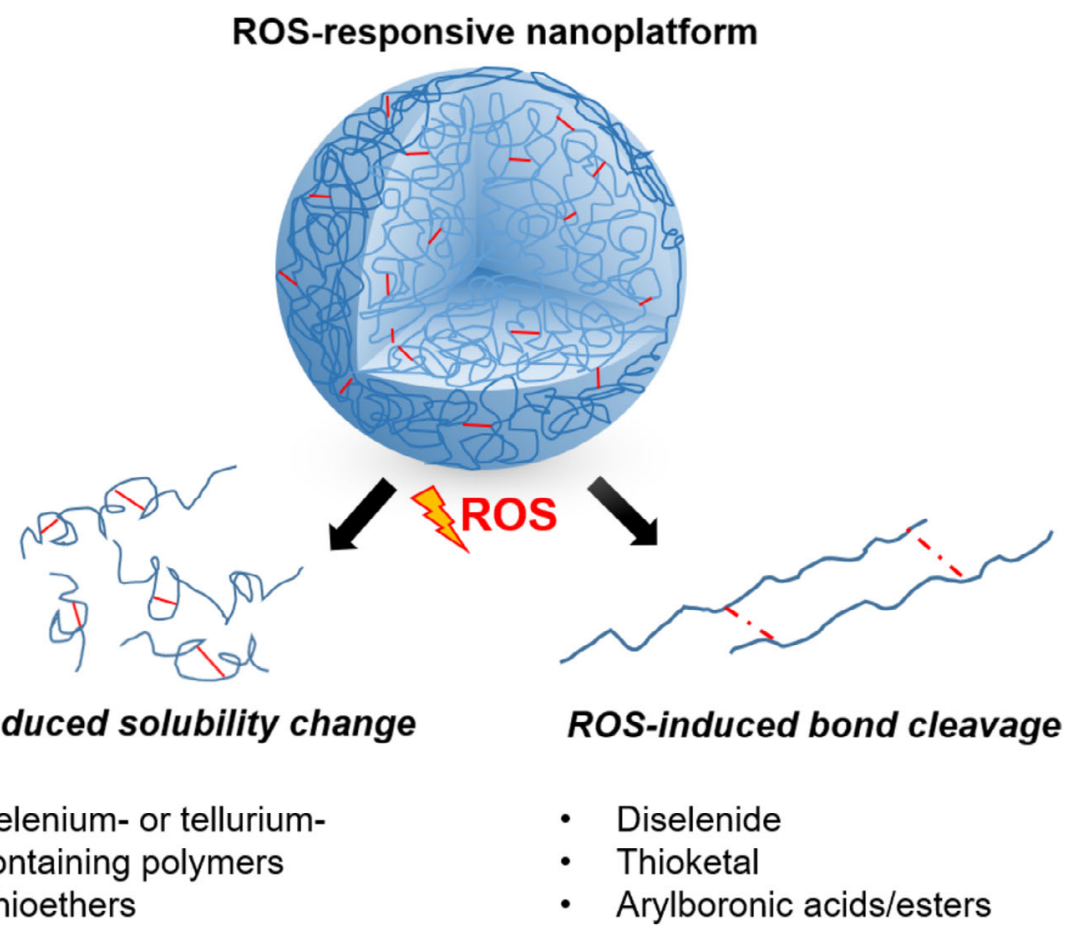

FIGURE 2 Schematic illustration of the possible behavior of ROS-responsive nanoplatforms in the presence of ROS. Polymer chains are indicated as blue lines; chemical bonds of the polymer are represented as red segments

\section{ROS-induced solubility change}

- Diselenide

- Thioketal

- Arylboronic acids/esters media. The second category consists of polymers that undergo backbone breakdown by cleavage of bonds sensitive to ROS. ${ }^{[18,19]}$ In addition to their potential of targeting and on-demand drug delivery, if ROS molecules are "consumed" in the reaction, these systems can also be explored for their ability to scavenge the excessively generated ROS and return ROS levels to normal levels. ${ }^{[22]}$

ROS-responsive materials drug delivery applications mainly possess limitations in: (a) the toxicity of the original polymer and its degradation products and (b) the ability to respond to ROS levels that are not physiologically relevant. However, the great variety of possible chemical structures and properties of ROS-responsive polymers represents a strong driving force to advance in the synthesis of new oxidation-responsive materials with improved biocompatibility and physiologically relevant responses.

\section{4 | ROS-RESPONSIVE NANOPLATFORMS FOR GIT DRUG DELIVERY}

Among the different diseases involving the GI tract, Crohn's disease and ulcerative colitis, the two main clinical forms of IBD, have been the protagonist of numerous studies, proposing innovative treatments and new delivery approaches. IBD is a chronic inflammatory disorder of the Gl tract affecting millions of patients around the world. ${ }^{[23]}$ Different strategies and diverse drugs have been developed for IBD therapy comprising of nonspecific agents, such as nonsteroidal antiinflammatory drugs and corticosteroids, as well as immunosuppressive and immunoregulatory agents. ${ }^{[24]}$ Precisely because of their non-specificity, the actual treatments generally lead to significant side effects that can reach severe forms, such as lower GI bleeding from a variety of sources, including diverticular disease, colitis and polyps, and they can also induce colitis in a previously normal bowel. ${ }^{[25]}$

An emerging opportunity to solve this problem is represented by nanotechnology and its therapeutic application, referred to as nanomedicine, which has been used for the development of orally administered dosage formulations designed as strategies to enhance uptake into diseased tissue within the colon or other parts of the GI tract. ${ }^{[15]}$ Nanoplatforms have the potential to directly access the intestinal mucosa and achieve a site-specific drug delivery. During the last 50 years, nanoparticles with different compositions, sizes, surface charges and targeting ligands have been investigated as carriers for drug delivery to inflamed intestinal areas. ${ }^{[26]}$ Nanoparticles with small size (below $200 \mathrm{~nm}$ ) accumulate in the areas of intestinal diseases compared to the healthy $\mathrm{GI}$ tissues, ${ }^{[27]}$ as a consequence of the compromised barrier function and increased particle uptake by macrophages. Nano-sized drug carriers could also display a prolonged intestinal transit time due to increased adhesion to the thick mucus layer. ${ }^{[28]}$ Nanomedicine, compared to conventional drugs, may also protect the payload from destabilization or hydrolysis, improving the bioavailability and increasing drug release/retention at diseased sites. $^{\text {[29] }}$

To overcome the multiple problems of IBD and other pathologies involving specific $\mathrm{Gl}$ areas, stimuli-responsive drug delivery systems represent a promising strategy due to their site-specific release ability in the presence of a stimulus associated with disease characteristics, such as different $\mathrm{pH}$ or ROS overproduction. ${ }^{\text {[30] }}$

Driven by the need for on-demand drug delivery, nanomedicines can be successfully combined with innovative stimuli-responsive materials (i.e., ROS-responsive) to develop smart nanoplatforms for targeted drug delivery to diseased tissues. ${ }^{[15]}$ Nanocarriers prepared with ROS-responsive materials (ROS-responsive drug delivery 
systems) are designed to release the therapeutic payload only at the site that produces excessive ROS (i.e., IBD) to reach both enhanced therapeutic efficiency and reduced side effects. ${ }^{[31]}$ Moreover, these ROS-responsive materials can be combined with other polymers, for example, polysaccharides, to increase the bioadhesion and biodegradability of the system to further improve the targeting activity ${ }^{[32]}$ and/or $\mathrm{pH}$-sensitive polymers to protect the system from the harsh gastric conditions orally administered formulations face.

In the next section we will discuss examples of ROS-responsive nanosystems for the treatment of GIT diseases.

\section{1 | Production techniques and characterization of ROS-responsive nanoplatforms}

Production techniques for ROS-responsive NPs were mostly based on self-assembling of the material with classical nanoprecipitation. In the case of Bertoni et al. ${ }^{[33]}$ and Li et al., ${ }^{[34]}$ a microfluidic approach was subsequently used to achieve an effective coating of the NPs with a $\mathrm{pH}$-responsive polymer to obtain the final microparticles formulation.

In the case of ROS-responsive nanoplatforms, the drug release behavior should be investigated in the presence of oxidative molecules (i.e., hydrogen peroxide) at a relevant concentration present in the oxidative environment of the inflamed colon. In another technique, GSH is typically added to the release solution to mimic the physiological reductive processes that happen during inflammation. After demonstrating the ROS-responsive release of the payload, in vitro and in vivo studies with a specific model that mimics the GIT, or the IBD should be performed. For example, in vitro, the intestinal membranes can be simulated with a co-cultured monolayer model, human colorectal adenocarcinoma cells Caco-2 and mucus secreting intestinal epithelial cells HT29-MTX, which form together TJs that mimic the in vivo intestinal epithelium ${ }^{[33,35-39]}$ to perform permeability studies.

In vivo studies use well established IBD affected mice models by inducing the inflammation with dextran sodium sulphate or TNBS in ethanol. ${ }^{[40]}$ In these models, biocompatibility, biodistribution and effective pharmaceutical efficacy of the prepared systems can be explored and evaluated.

Table 1 summarizes some of characterization techniques and model used commonly in stimuli-responsive nanoplatforms development for inflamed intestinal diseases.

\section{2 | Types of ROS-responsive nanoplatforms}

Progress in polymer chemistry has led to the development of several novel ROS-responsive biomaterials in the last few years, which have provided specific sensitivity toward $\mathrm{H}_{2} \mathrm{O}_{2}$ as a therapeutic approach for oxidation-sensitive systems. ${ }^{[47]}$

Taking advantage of the increased ROS concentration in diseased tissue in IBD, Wilson et al. ${ }^{[44]}$ developed a new poly-(1,4-phenyleneacetone dimethylene thioketal) (PPADT) ROS-sensitive polymer for targeting inflamed intestinal tissues that were stable in

TAB LE 1 Characterization techniques and models for stimuli-responsive nanoplatforms developed for GIT drug delivery

\begin{tabular}{|c|c|c|c|}
\hline Technique & Type & Use & References \\
\hline ROS-responsive drug release & In vitro & $\begin{array}{l}\text { In vitro drug release tests in presence of } \mathrm{H}_{2} \mathrm{O}_{2} \text { as } \\
\text { reducing agent to assess the ROS-responsive } \\
\text { behavior of the system. }\end{array}$ & {$[33,41,42]$} \\
\hline Mucin adsorption & In vitro & $\begin{array}{l}\text { An in vitro colorimetric method to predict the mucin } \\
\text { adsorption of the nanosystem in the intestine. }\end{array}$ & {$[42,43]$} \\
\hline C2BBe1/HT29-MTX cell monolayer & In vitro & $\begin{array}{l}\text { In vitro model forming tight junctions and secreting } \\
\text { mucus to mimic the in vivo intestinal epithelium. } \\
\text { Useful for permeability studies. }\end{array}$ & {$[33-35]$} \\
\hline Myeloperoxidase activity & In vitro & $\begin{array}{l}\text { Useful for the evaluation of inflammation also in } \\
\text { inflammatory bowel disease }\end{array}$ & {$[41,42,44]$} \\
\hline DSS induced colitis & In vivo & $\begin{array}{l}\text { This model can be used in mice, rats, hamster or } \\
\text { guinea-pigs inducing an acute colitis characterized } \\
\text { by bloody diarrhea, ulcerations and infiltrations with } \\
\text { granulocytes. It is particularly useful to study the } \\
\text { contribution of innate immune mechanisms of colitis. }\end{array}$ & {$[40-42,44]$} \\
\hline TNBS induced colitis & In vivo & $\begin{array}{l}\text { This model is useful to study } T \text { helper cell-dependent } \\
\text { mucosal immune responses. It is required particularly } \\
\text { to generate chronic colitis characterized by a } \\
\text { predominant Th1-mediated immune response with } \\
\text { dense infiltration of lymphocytes/macrophages and } \\
\text { thickening of the colon wall. }\end{array}$ & {$[40,45,46]$} \\
\hline Oxazolone induced colitis & In vivo & $\begin{array}{l}\text { This model induces a severe colitis in rats or mice, } \\
\text { characterized by weight reduction, diarrhea and } \\
\text { marked loss of globet cells. It is in some aspects } \\
\text { similar to characteristics observed in human } \\
\text { ulcerative colitis. }\end{array}$ & {$[40]$} \\
\hline
\end{tabular}


acidic, basic and protease-abundant environments analogous to the GI tract. Using PPADT, they formulated ROS-responsive nanoparticles (TKNs) via an oil-in-water single-emulsion procedure. TKNs degraded when reaching the site of intestinal inflammation via thioketal linkages, thus localizing the release of the payload to inflamed intestinal tissue.

PPADT was used to encapsulate TNF- $\alpha$ siRNA complexed with the cationic lipid, 1,2-dioleoyl-3-trimethylammonium- propane (TNF- $\alpha$ -DOTAP), to form nanoparticles (TNF- $\alpha-$ TKNs) for subsequent in vitro and in vivo studies with mice suffering from dextran sodium sulphate (DSS)-induced colitis. ${ }^{[40]}$ Complexing siRNA with cationic species (i.e., DOTAP) enhanced siRNA transfection by increasing siRNA stability, ${ }^{[48]}$ mucosal transport, ${ }^{[49]}$ cellular internalization, ${ }^{[50]}$ and endosomal escape. ${ }^{[51]}$ Furthermore, the incorporation of DOTAP gave the nanoparticles a positive surface charge that increased particle uptake by phagocytes ${ }^{[52]}$ and adhesion to the negatively charged intestinal mucosa. ${ }^{[53]}$ The siRNA-loaded TKNs (TNF- $\alpha$-TKNs) were optimized to have a diameter of around $600 \mathrm{~nm}$ to limit the nonspecific uptake by enterocytes ${ }^{[54]}$ and improved the uptake by phagocytes, which are the main producers of TNF- $\alpha$ at sites of intestinal

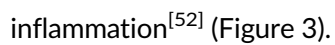

In vitro studies using lipopolysaccharide (LPS)-activated macrophages showed a significant reduction in TNF- $\alpha$ production when treated with TNF- $\alpha-T K N$ s compared to cells treated with either phosphate buffered saline (PBS) or TKNs loaded with scrambled siRNA sequence. Subsequent in vivo studies using mice suffering from DSSinduced colitis demonstrated the ability of TNF- $\alpha-T K N$ s to effectively target inflamed intestinal tissues and suppress mRNA levels of TNF- $\alpha$ and several other pro-inflammatory cytokines (IL-1, IL-6 and IFN- $\gamma$ ). Other systems, such as TNF- $\alpha$-siRNA loaded PLGA nanoparticles and TNF- $\alpha$-DOTAP were used as a control and demonstrated characteristics associated with DSS-induced inflammation, including high levels of myeloperoxidase activity and significant weight loss. These results support the ability of TKNs to target inflamed tissues, as an important factor for their in vivo efficacy.

These positive results indicated successful siRNA delivery to the inflamed intestinal tissues through the highly ROS-specific response of the TKNs and, moreover, they confirmed that this ROS-responsive material with a high stimulus specificity can withstand the harsh environments of the $\mathrm{Gl}$ tract and serve as a vehicle for successful oral delivery of therapeutic agents in IBD with a pathophysiological level of oxidative stress.

(a)

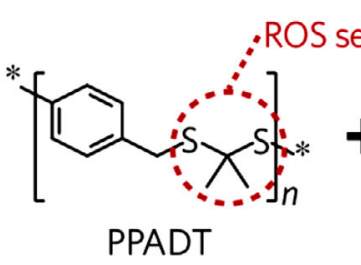
PPADT

(b)

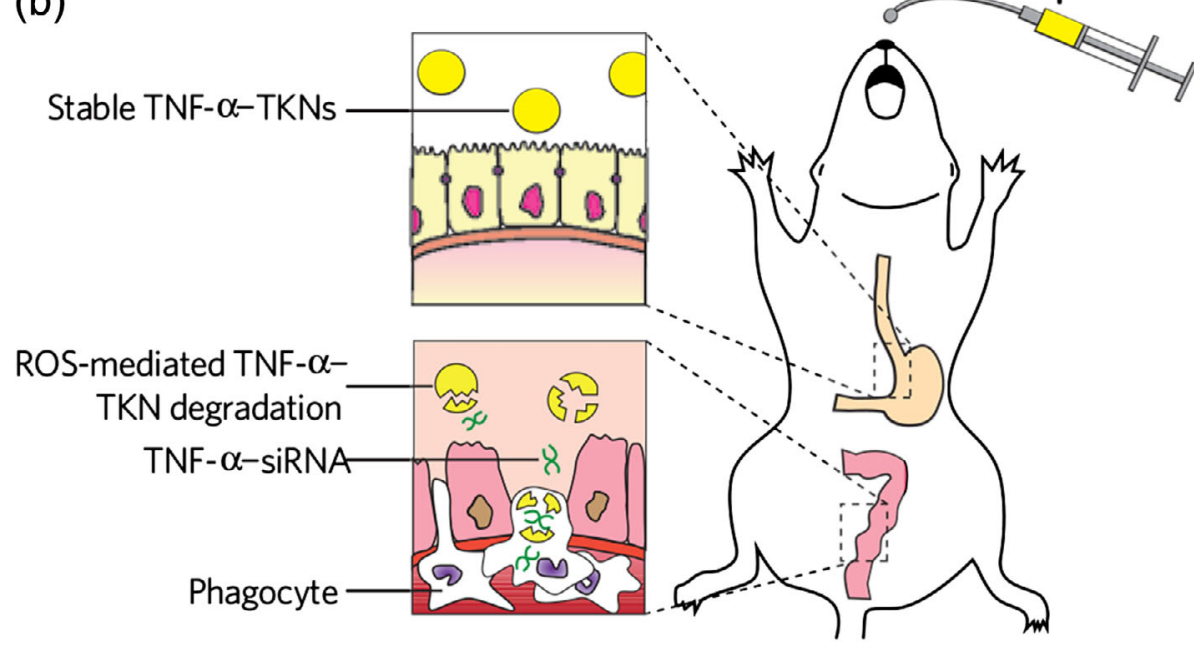

(c)<smiles>COC(C)(C)OC(C)(C)OC</smiles>

1

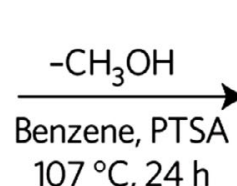

2 $107^{\circ} \mathrm{C}, 24 \mathrm{~h}$

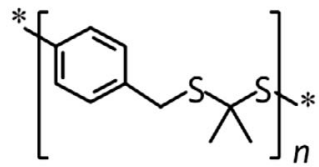

3
FIGURE 3 Thioketal nanoparticles are formulated from a ROS-sensitive polymer and release orally delivered siRNA at sites of intestinal inflammation. (a), PPADT 3 is a new polymer composed of ROS-sensitive thioketal linkages (circled dashed). TNF$\alpha-$ TKNs were prepared by first precomplexing TNF- $\alpha$-siRNA with the cationic lipid DOTAP. Next, these TNF- $\alpha-$ DOTAP complexes were added to an organic solution containing PPADT. The scanning electron micrograph shows TNF- $\alpha$-TKNs (scale bar represents $1.5 \mu \mathrm{m})$. (b), When delivered orally, TNF$\alpha-$ TKNs remain stable in the harsh environment of the gastrointestinal tract, protecting TNF- $\alpha-$ siRNA and preventing its release to non-inflamed mucosal tissues. However, at sites of intestinal inflammation, where infiltrating phagocytes produce unusually high levels of ROS, the TKNs degrade, thus releasing TNF- $\alpha-$-siRNA to the site of inflammation. (c), PPADT 3 was synthesized using the acetal exchange reaction. PTSA: paratoluenesulphonic acid. Reproduced with permission from Reference [44]; (c) 2010 Springer Nature Limited 
FIGURE 4 Design, preparation, and characterization of a SOD/catalase mimetic nanomedicine. Composition and engeneering of a SOD/catalase mimetic nanomedicine Tpl/OxbCD NP (a). Targeted therapy of colitis by Tpl/OxbCD NP (b). Reproduced with permission from Reference [41]; (C) 2016 Elsevier B.V
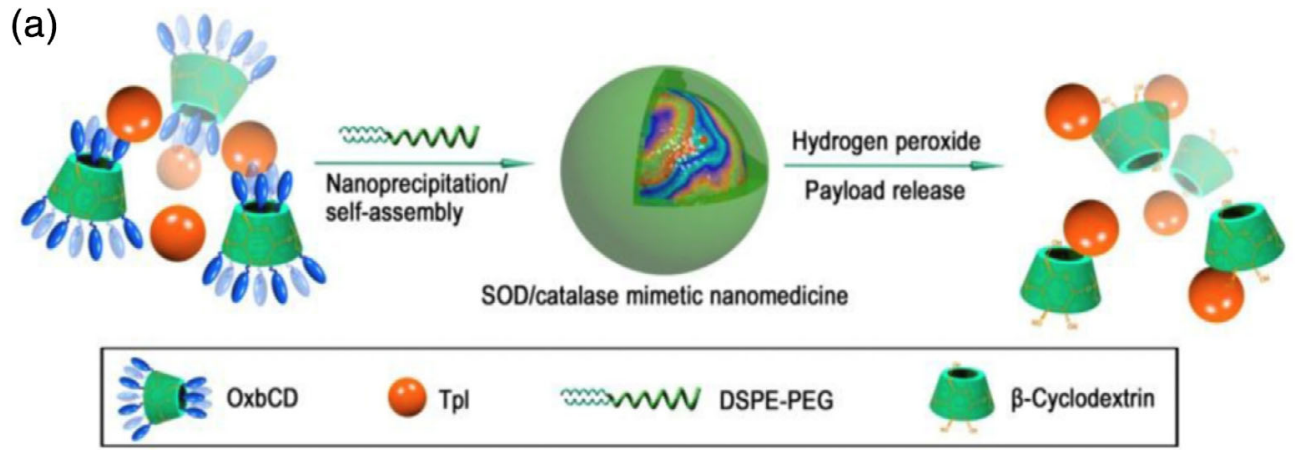

(b)

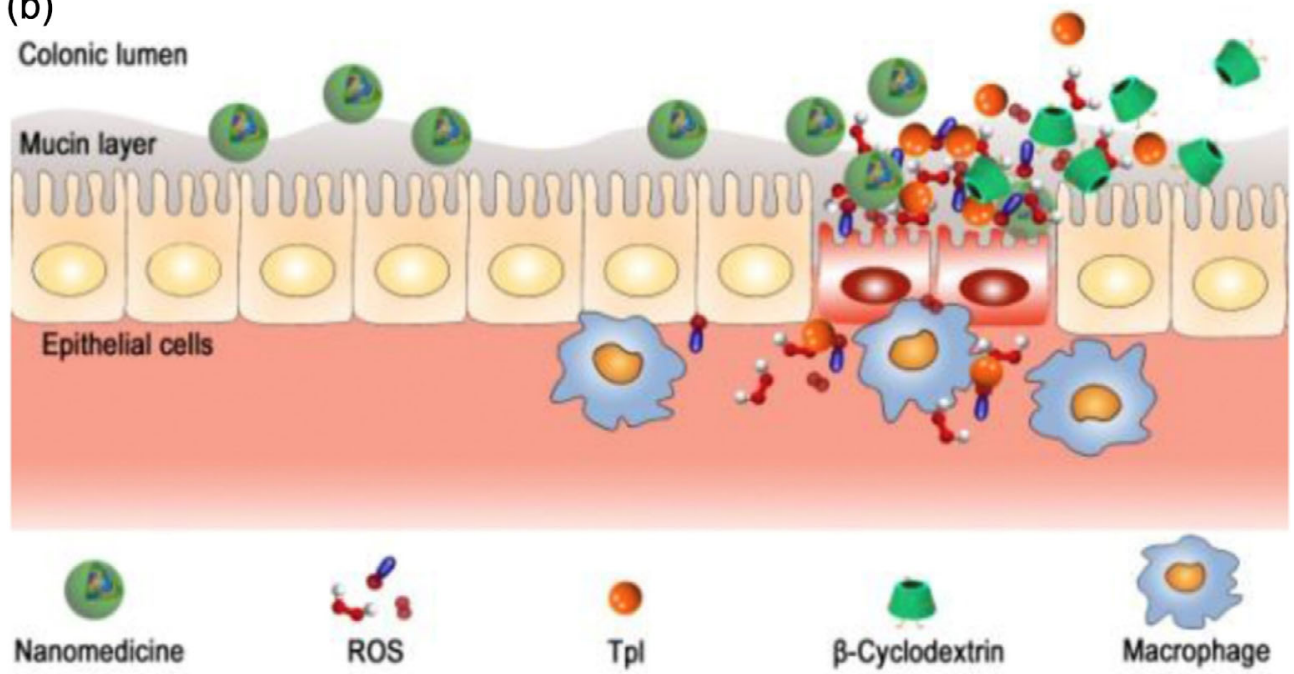

Zhang et al. ${ }^{[41]}$ also developed a nanosystem based on Tempol (Tpl selected as a SOD-mimetic drug) loaded in a biocompatible ß-cyclodextrin-derived material (OxbCD), as shown in Figure 4. This nanomedicine (Tpl/OxbCD NP), prepared by a nanoprecipitation/self-assembly method, was stable in the GI tract and could target inflammatory sites by releasing the payload in the presence of non-physiologically high ROS levels. OxbCD was synthesized by functionalization of $\beta-C D$ with an oxidation-labile moiety of 4-(hydroxymethyl) phenylboronic acid pinacol ester (PBAP). Its ROS-responsive hydrolytic profile was assessed, confirming that OxbCD NPs were completely hydrolyzed in the presence of $1 \mathrm{mM}$ of $\mathrm{H}_{2} \mathrm{O}_{2}$ within 2 hours. Furthermore, OxbCD NPs may stoichiometrically eliminate $\mathrm{H}_{2} \mathrm{O}_{2}$ (catalase-mimicker behavior) that concomitantly with $\mathrm{OxbCD}$ hydrolysis, is transformed into water. The stability at different $\mathrm{pH}$ values covering the entire $\mathrm{pH}$ spectrum of the $\mathrm{Gl}$ tract $(1.2,6.8$, and 7.4$)$ was confirmed up to 300 minutes. The release profiles of Tpl/OxbCD NP was evaluated in vitro in various buffers including PBS pH 7.4 and 1.2 with or without the presence of $1 \mathrm{mM}$ of $\mathrm{H}_{2} \mathrm{O}_{2}$ for 6 hours, demonstrating that the payload release was highly responsive to $\mathrm{H}_{2} \mathrm{O}_{2}$ level.

After in vitro characterization, the selective accumulation in the inflamed colon of OxbCD NP was proved first ex vivo and then in vivo, using mice suffering from DDS-induced colitis, using PLGA nanoparticles as control. Then, therapeutic effects of Tpl/OxbCD NP in DSS-induced acute colitis in mice was explored treating the animals for 7 days with daily oral administration of Tpl alone, Tpl loaded PLGA nanoparticles and Tpl/OxbCD nanoparticles. The body weight of all DSS-treated mice gradually decreased except for the animals treated with Tpl/OxbCD nanoparticles, which had a minimal weight loss. Moreover, Tpl/OxbCD nanoparticle-treated mice displayed much lower disease activity and wellpreserved colonic length compared to model mice. The ROS-responsive formulation also showed reduced levels of inflammatory cytokines, such as TNF- $\alpha$, INF- $\gamma$ and IL-8ß, and a significant decrease in CD98 (a type II transmembrane protein that may promote the development and progression of $\mathrm{IBD}^{[55]}$ ) expression, confirming the effective therapeutic effect of $\mathrm{Tpl} / \mathrm{OxbCD}$ nanoparticles. Finally, the same formulation was tested in mice with TBNS-induced colitis ${ }^{[40]}$ and showed effective inflamed colon targeting and minimal weight loss after treatment.

With another innovative material synthesis, Sun et al. ${ }^{[42]}$ developed an oral BDS (a synthetic glucocorticoid with high topical antiinflammatory activity, but limited systemic activity due to high first-pass metabolism in the liver ${ }^{[56]}$ ) nanocarrier system with therapeutic potential for IBD. An amphiphilic inulin derivative was synthesized from the carboxymethyl inulin (CMI), bearing carboxyl groups in the side chain. Then, 4-aminothiophenol (ATP) was introduced to CMI by forming an amide bond, using 1-ethyl-3-(3-dimethylaminopropyl)carbodiimide (EDC)mediated chemistry, producing a 4-aminothiophenol-carboxymethyl inulin 
conjugate (ATP-CMI). The nanoparticles were prepared by selfassembling of the ATP-CMI followed by sonication for 6 minutes to facilitate the oxidation reaction of thiol groups. In vitro stimuliresponsive release showed that small amounts of BDS were released in acidic and neutral conditions, while more than $80 \mathrm{wt} \%$ of the payload was released when the drug-loaded nanoparticles were exposed to $\mathrm{pH} 6.0$ in the presence of glutathione (GSH), which was used as a reducing agent to mimic physiological reductive processes that occur during ROS overproduction in the inflammation sites. ${ }^{[57]}$ These results demonstrated that the oxidation-responsive nanoparticles can efficiently transport the payload until reaching the inflamed colon tissue and subsequently release the drug for effective targeted activity. The nanoformulation presented in vitro cytocompatibility when incubated with Caco-2 cells with NPs concentrations ranging from 50 to $500 \mu \mathrm{g} \mathrm{mL}^{-1}$ for 24 hours.

The effective therapeutic efficacy was established using DSSinduced IBD mice where decreased inflammation, lower levels of IL-6 and TNF- $\alpha$, and a higher level of IL- 4 were observed in mice treated with the oxidation-responsive BDS-nanoparticles compared to BDS alone and PBS controls. The enhanced therapeutic efficacy of BDSnanoparticles in colitis was due to: (i) their selective adhesion to inflamed segments, because of the negative surface charge of ATPCMI nanoparticles; (ii) the formation of disulfide bond with the intestinal mucosa that can prolong the residence time of the delivery system in the inflammatory sites; (iii) the redox-sensitive release of BDS based on the reduction-oxidation processes of thiol groups on ATP$\mathrm{CMI}$ nanoparticles achieve a higher drug concentration gradient at the absorption sites facilitating drug absorption.

With the aim of release cargo from nanoparticles in the presence of biologically relevant levels of hydrogen peroxide, De Gracia Lux et al. ${ }^{[58]}$ synthesized two innovative polymers presenting boronic ester groups in the backbone, one directly linked and the other one linked via an ether linkage. Both were formulated into nanoparticles with a size of approximately $150 \mathrm{~nm}$ via an oil/water emulsion and loaded with a fluorescent dye (Nile red) as model drug. Interestingly, the second polymer presented a degradation about an order of magnitude compared to the first one, releasing $50 \%$ of the payload after 6 hours of exposure to $100 \mu \mathrm{M} \mathrm{H}_{2} \mathrm{O}_{2}$. The payload release of NPs formulated with this polymer was also tested with and without activating ROS production in neutrophils demonstrating a 2-fold increased release in presence of oxidative environment compared to PLGA NPs and a control polymer similar in structure with the synthesized one. The ROS-responsive property of this new polymer can be effective for an application in targeting inflamed gastrointestinal tract.

\section{5 | FROM ROS-RESPONSIVE MATERIALS TO MULTI-STIMULI RESPONSIVE APPROACHES TO TARGETED COLONIC DRUG DELIVERY}

Various pathological abnormalities in IBD are exploited to develop stimuli-responsive drug delivery systems with high therapeutic localization at the inflamed intestinal site and limited adverse reactions. ${ }^{[15,47,59-61]}$ These stimuli are categorized by whether the delivery system interacts with or is actuated by internal biological signals or external means controlled remotely from the body. Internal stimuli, such as deviations in $\mathrm{pH}$ and elevated ROS levels in diseased tissues, has been widely used to overcome physiological barriers in drug delivery, as previously discussed. ${ }^{[33,34,41,44,57,59,62]}$ While single stimuli-responsive systems have demonstrated some success in protecting the therapeutic cargo from acidic $\mathrm{pHs}$ in the stomach or sustaining the cargo release in the presence of enzymes associated with IBD, they have also led to unspecific and premature drug release due to the multiple physiological barriers present during the therapeutics' trajectory to the colon. ${ }^{[63-65]}$

Multi-stimuli responsive drug delivery vectors are advantageous over single response carriers, because they protect the drug cargo while simultaneously or subsequently controlling drug release over various physiological barriers that are presented as the carrier moves through the GIT. ${ }^{[66,67]}$ The $\mathrm{pH}$ responsive systems are typically used to protect cargo from the acidic stomach ( $\mathrm{pH}$ 1.2), but colon targeting may remain problematic after the $\mathrm{pH}$ responsive coating is degraded in the stomach. For example, ulcerative colitis treatment should occur in the distal colon and rectum, ${ }^{[68]}$ but the commercially available single $\mathrm{pH}$-responsive budesonide products Budenofalk and Entocort demonstrated premature drug release in the ileum, which has a $\mathrm{pH}$ resembling that of the colon. ${ }^{[69]}$ This drug release in the ileum, thus prevented the sufficient delivery of therapeutics to inflamed areas in the distal colon. By having a multi-stimuli responsive system, more specifically a hierarchal stimuli-responsive structure where different stimuli are subsequently actuated, the cargo begins $\mathrm{pH}$-mediated degradation upon reaching the ileum and large intestine region, followed by another stimuli-responsive layer triggering upon reaching the colon. This approach delivers the system from the small intestine to the colon with minimal cargo release, thus improving site specificity and drug distribution in the colon.

The following section will discuss examples of dual-responsive drug delivery systems, including $\mathrm{pH}$ with $\mathrm{ROS}$, and enzymatic responses, emerging external stimuli systems, and how these external systems can be used in conjunction with internal stimuli to further improve on therapeutic distribution in the colon. Finally, we will discuss other targeting strategies used for simultaneously stimuliresponsive carriers for IBD treatment.

\section{1 | $\mathrm{pH}-$ Mediated dual-responsive drug delivery carriers}

As previously mentioned, $\mathrm{pH}$-responsive coatings are critical for protecting the therapeutic cargo from acidic conditions in the stomach, but an additional protective layer is needed to sustain the cargo release as it travels through the large intestine, where the $\mathrm{pH}$ resembles that of the colon. ${ }^{[70]}$ Multi-compartmental or hierarchal nano-inmicro (NiMOS) structures with dual $\mathrm{pH}$ and enzyme, bacteria, or ROS responsive materials were developed to show increased therapeutic targeting to the colon. ${ }^{[14,53,54]}$ While there are a multitude of 
examples that demonstrate dual $\mathrm{pH}$ and other stimuli responsive carriers for drug delivery to the colon, as the following section will discuss, there remains limited research on combining $\mathrm{pH}$ and ROSresponsive carriers for colitis-related treatments. This section will discuss dual-responsive stimuli systems that have been developed for drug delivery to the inflamed colon to understand potential carrier materials and designs that can be combined with ROS-responsive systems in future studies. By exploring other dual-responsive systems, the reader may find useful considerations for developing dualresponsive ROS-mediated nanocarriers with applications in inflamed colonic drug delivery and inspire their application in dual pH-enzyme responsive systems for targeted IBD therapy. Although the focus of this review is on ROS-responsive systems, it is pertinent to explore other dual-responsive systems from, which we can apply to ROSmediated nanocarriers.

\subsection{1 $\mathrm{pH}$ and enzyme responsive systems}

When designing enzyme responsive drug delivery systems, it is important to consider the intestine microflora or what enzymes are present in the different components of the Gl tract and at what concentrations. For example, the lumen of the small intestine contains gram quantities of trypsin, chymotrypsin, and cathepsin- $\beta$, which are all secreted from the pancreas. ${ }^{[71]}$ Moreover, matrix metalloproteinase (MMP) and esterases enzymes are upregulated in IBD and other inflammatory conditions. ${ }^{[72-74]}$ Patients with ulcerative colitis demonstrated significantly higher levels of MMPs in the inflamed mucosa of the distal colon and non-involved mucosa of the proximal colon. ${ }^{\text {[2] }}$ Thus, these enzymes serve an opportunity for therapeutic targeting in the inflamed colon via enzyme-responsive carriers. Ideally, the carrier should continue protecting the cargo throughout the small and large intestine until it reaches the distal colon where drug release is mediated by the presence of MMPs.

Small molecules with MMP-labile bonds, such as triglycerol monostearate and acorbyl palmitate (AP), were used to form dual-pH, enzyme-responsive hydrogels in Li et al. ${ }^{[34]} \mathrm{BDS}$, a glucocorticoid for
IBD therapy, and AP were co-loaded inside hyaluronic acid functionalized porous silicon (PSi) based nanoparticles (PSi-HA). The nanoparticles were subsequently encapsulated by hydroxypropyl methylcellulose acetate succinate (HPMCAS), a $\mathrm{pH}$-responsive material, to form the final dual-responsive hierarchal structured drug delivery system AP@PSi-HA@HPMCAS. Figure 5 shows the schematic for producing this hierarchal stimuli-responsive structure.

Release profiles mimicking the $\mathrm{pH}$ gradient in the GIT showed minimal release under acidic stomach conditions, and thus, the $\mathrm{pH}$ responsive matrix protected the embedded enzyme-responsive composite. Upon addition of the enzyme lipase at $\mathrm{pH} 5$, a lipase concentration-dependent release is observed. This tailored drug release would be particularly useful for modulating the drug concentration in the colon by disease severity. Specifically, for IBD with higher extent of inflammation and inflammation-associated enzyme production, higher therapeutic amounts would be released into the colon.

While this work demonstrated a sophisticated way to overcome multiple physiological barriers as the therapeutic travels from the oral cavity to colon, immunofluorescence images were only conducted for the distal end of the colon and it is pertinent to observe the fluorescence in other GIT components, specifically those with similar $\mathrm{pH}$ to that of the colon, dissected from mice with colitis. Naeem et al. ${ }^{[46]}$ measured the in vivo localization of the model drug Coumarin-6 (C-6) in different GIT components to demonstrate the advantages of a dual $\mathrm{pH}$-enzyme-responsive drug delivery system. The dose amount was measured in the stomach, small intestine, cecum, and colon, and similar dose levels were found in the stomach and small intestine for $\mathrm{pH}$ coated and dual coatings. Within the cecum and colon there was a 2.5- and 5.5-fold dose increase for $\mathrm{pH}$ - and dual $\mathrm{pH}$-enzymeresponse systems. This system was subsequently applied to enhance the therapeutic efficacy of BSD in ulcerative colitis. ${ }^{[45]}$

The aforementioned dual $\mathrm{pH}$ - and enzyme-responsive drug delivery systems were also monitored for reduction in inflammation biomarkers commonly associated with IBD, such as IL-1 $\beta$, IL-6, and TNF$\alpha^{[15,75]} \mathrm{Li}$ et al. ${ }^{[34]}$ showed a 5 -fold decrease in IL-6 for different

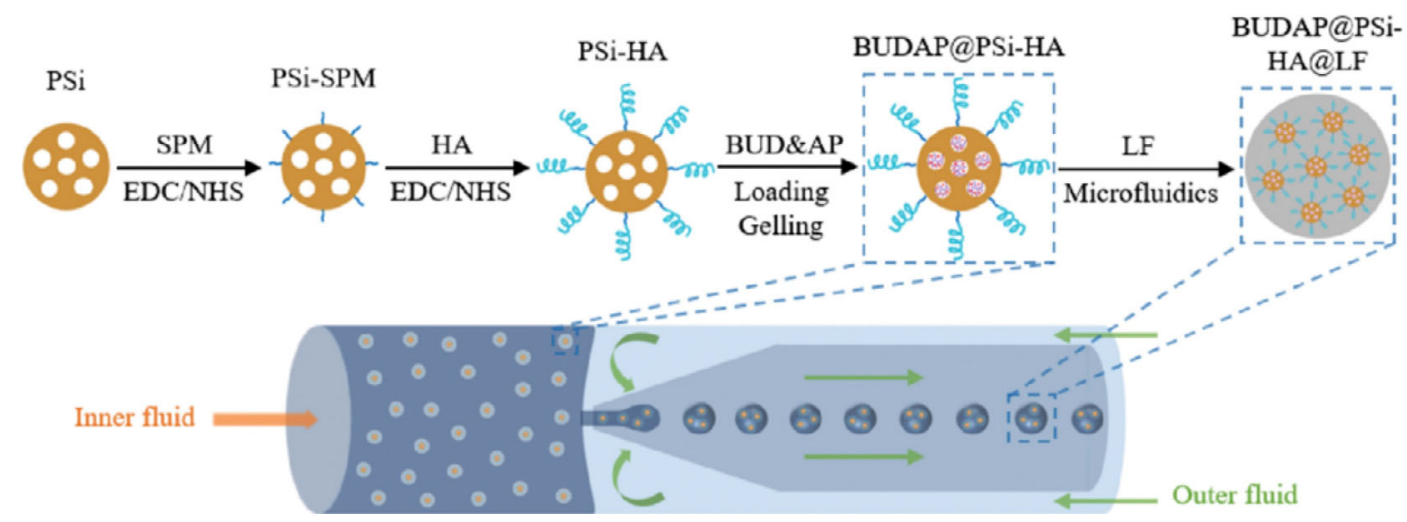

FIGURE 5 Schematic for fabricating AP@PSi-HA@HPMCAS hierarchal nano-in-microparticles. Hyaluronic-functionalized porous silicon nanoparticles are co-loaded with budesonide and the enzyme-responsive acorbyl palmitate (AP). The AP is gelled to lock BUD inside the PSi. AP@PSi-HA is subsequently encapsulated with the $\mathrm{pH}$ responsive HPMCAS by a microfluidics approach to produce the final structure. Reproduced with permission from Ref. [34] (CC BY-NC 4.0); ( 2018 Elsevier B.V 
formulations in comparison to the control that did not receive any treatment. However, only in IL-1 $\beta$ was there a significant reduction between single $\mathrm{pH}$-responsive systems and the dual $\mathrm{pH}$ - and enzymeone. Naeem et al. also observed the most significant TNF- $\alpha$ reduction in the dual $\mathrm{pH}$ - and enzyme-responsive formulation. ${ }^{[45]}$

The TNF- $\alpha$ knockdown was also a key strategy for the oral delivery of siRNA, using dual $\mathrm{pH}^{-}$and enzyme-responsive microencapsulated nanogels. ${ }^{[76,77]}$ Oral delivery of siRNA presents an additional challenge, because these gene-silencing molecules undergo degradation by enzymes and harsh gastric conditions. Moreover, intracellular delivery and endosomal escape must be achieved, while maintaining the siRNA integrity. siRNA was loaded into polycationic 2-(diethylamino)ethyl methacrylate (DEAEMA)-based nanogels, which were previously shown to facilitate intracellular delivery and endosomal escape. The polycationic nanogels were then encapsulated with an enzymatically and $\mathrm{pH}$ degradable hydrogel. Therefore, after protection in gastric conditions followed by degradation in intestinal conditions, the nanogels can help facilitate accumulation in the inflamed intestinal tissue, where phagocytotic macrophages are present. The nanogels and degraded microgels both showed a significant reduction in TNF- $\alpha$ expression in comparison to a control siRNA. Moreover, non-degraded microgels did not induce TNF- $\alpha$ knockdown, indicating the protective capabilities of the microgel platform.

Clinton et al. also explored the material properties, such as microparticle polymer crosslinking density, on the oral delivery capability of commercially available therapeutic proteins, such as Rituxan. ${ }^{[77]}$ Such strategies and material systems can potentially be applied to therapeutic proteins used to treat Crohn's disease, such as Adalimumab and Infliximab. While $\mathrm{pH}$ - and enzyme-responsive drug delivery systems pose efficient strategies for overcoming the multiple physiological barriers faced by therapeutics as they travel through the GIT, other physiological abnormalities, such as elevated ROS levels, can be also exploited to increase the therapeutic concentration at the diseased site of interest.

\subsection{2 $\mathrm{pH}$ and ROS responsive systems}

Reactive oxygen metabolites are inflammatory mediators that play an essential role in the pathogenesis and progression of IBD, as previously discussed. While there have been a variety of drug delivery examples that exploit the excessive generation of ROS as a diseasespecific triggering mechanism in inflammation-related diseases, such as IBD and cancer, there are limited systems in the literature demonstrating a dual-responsive therapeutic release.

As mentioned previously, a dual-stimuli-responsive system triggered by $\mathrm{pH}$ and subsequently enzyme or ROS-mediated degradation can further enhance therapeutic efficacy, where the $\mathrm{pH}$ coating protects the carrier from degradation due to varying $\mathrm{pH}$ throughout the GIT. Upon reaching the inflamed site of interest, the therapeutic is released not only proximally, but also precisely to the inflamed tissue, where ROS levels are upregulated and not to surrounding healthy tissue. This section will discuss an example where such a dualresponsive carrier is used in the context of IBD, and the more prevalent literature on colon cancer applications. While colon cancer is a different application, there are similarities in physiological barriers and considerations that can be applied to future carriers developed for IBD.

Taking advantage of a sequential $\mathrm{pH}$ and $\mathrm{ROS}$ responsive behavior, Bertoni et al. ${ }^{[33]}$ formulated an innovative targeted nano-inmicro therapy for IBD. To achieve this result, an $\mathrm{H}_{2} \mathrm{O}_{2}$-responsive dextran (OxiDEX ${ }^{[78]}$ ) was synthesized and used to prepare nanoparticles by nanoprecipitation, using a microfluidics approach. The nanoparticles were subsequently coated with chitosan (CS), known for its mucoadhesive properties to add an advantage for IBD targeting. ${ }^{[15]}$ Rifaximin (RIF) was chosen as model drug (because it has successfully induced remission of $\mathrm{IBD}^{[79]}$ ) and effectively loaded in the nanoparticles (CS-RIF). The RIF loaded and CS coated nanoparticles were finally encapsulated by microfluidics in a $\mathrm{pH}$ responsive hydroxypropyl methyl-cellulose acetate succinate (HPMCAS) polymer to obtain the definitive micro-composite. The coating was added to protect the nanoparticles from the harsh conditions of the stomach and to release them in the inflamed intestine tissues, where an abnormal level of ROS is produced. Figure 6 shows a schematic for the preparation of this dual $\mathrm{pH}$-ROS-responsive carrier for targeting GIT delivery.

The stability of CS-RIF nanoparticles was confirmed in a medium simulating intestinal conditions and the oxidation-responsive degradation of NPs was confirmed with different concentrations of $\mathrm{H}_{2} \mathrm{O}_{2}$ from $100 \times 10^{-6}$ to $1.0 \times 10^{-3} \mathrm{M}$. Furthermore, the $\mathrm{pH}$-responsive behavior of the coating polymer was defined immerging the microparticles at $\mathrm{pH} 1.2$ in which the formulation remained unchanged, and then at $\mathrm{pH} 6.8$ in which the microparticles started to lose their shape after 5 minutes. This characteristic proved the ability of the final formulation to tolerate the harsh gastric conditions $(\mathrm{pH} 1.2)$ and to release the encapsulated nanoparticles only at the typical intestinal $\mathrm{pH}$ (6.8).

After assessing the in vitro cytocompatibility in two cell lines (C2BBe1 and HT29-MTX), the interaction between nanoparticles and intestinal cells monolayer in vitro model (C2BBe1/ HT29-MTX) was qualitatively studied by confocal laser scanning microscopy. This study confirmed the mucoadhesive properties of the nanoparticles due to the CS coating. Finally, using the in vitro cell monolayer model formed with a co-culture of C2bbe1/HT29-MTX that closely mimics the in vivo intestinal membrane, ${ }^{[35]}$ the drug permeability across membrane was evaluated confirming that in a high-oxidative extracellular environment, the payload release from the ROS-responsive nanoformulation was complete, and RIF was not released from OxiDEX NPs in healthy conditions, representing an advantage in terms of unspecific adsorption and systemic side effects. While this formulation demonstrated promising results in mitigating unspecific absorption and systemic side effects, future in vivo studies are needed to observe the therapeutic distribution and extent of inflammation reduction throughout the GIT.

Dual $\mathrm{pH}$-and ROS-responsive drug delivery carriers to the GIT have been more widely studied in colorectal cancer applications. ${ }^{[80,81]}$ 
(a)
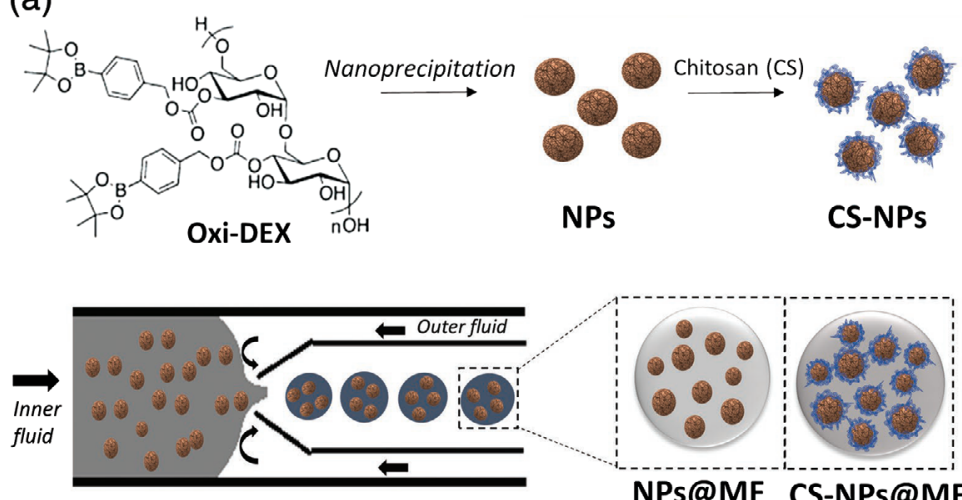

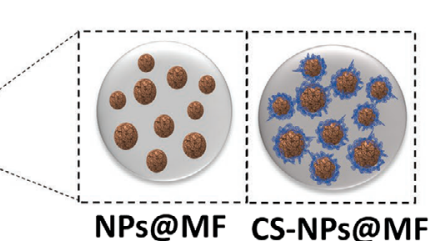

(b)

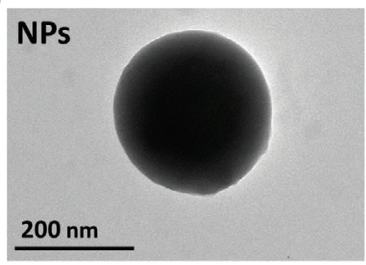

(c)

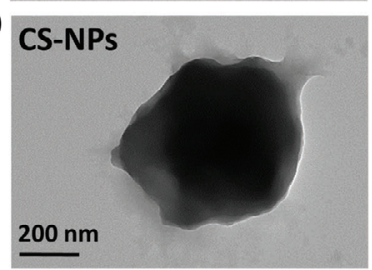

FIGURE 6 Schematic illustration of the production of nano-in-micro Oxi-Dex@CS@HPMCAS for Rifaximin delivery to the colon. (a), The ROS-responsive Oxi-dex nanoparticles are formed by nanoprecipitation and coated with chitosan (CS) before being encapsulated by the $\mathrm{pH}$ responsive polymer HPMCAS through a microfluidics approach. TEM images of (b), Oxi-dex NPs formed after nanoprecipitation and (c), Oxi-dex NPs coated with chitosan. Reproduced with permission from Reference [33]; (c) 2018 Wiley-VCH

For example, Lee et al. ${ }^{[80]}$ used redox-responsive nanoparticles with a diselenide linkage core cross-linked with ChitoPEG copolymers (ChitoPEGse) to deliver Piperlongumine (PL), a natural alkaloid extract from piper plants. Release studies were conducted between a $\mathrm{pH}$ of 5.8 and 7.4 to mimic the slightly acidic conditions present in the tumor microenvironment. The PL release rate and uptake by CT26 mouse colorectal carcinoma cells increased in physiological abnormalities present within the tumor microenvironment (i.e., acidic conditions and elevated ROS levels). However, these $\mathrm{pH}$ levels are, again, representative of a tumor microenvironment and not acidic gastric conditions. Therefore, it would be of interest to combine the redox-responsive component with $\mathrm{pH}$ responsive materials that are degraded at $\mathrm{pH}$ levels representing that of the GIT during oral drug delivery.

Other dual $\mathrm{pH}$-and ROS-responsive carriers for colorectal cancer therapeutic delivery have utilized zinc protoporphyrin (ZnPP)-loaded with a pH sensitive polymeric prodrug of benzoyloxycinnamaldehyde ${ }^{[82]}$ and the hybrid anticancer prodrug [4-(1,3, 2-dioxaborinan-2-yl)benzyl ([5-methyl-2-styryl-1,3-dioxan5-yl] methyl) carbonate], which coupled a quinone methide-generating moiety to a ROS-generating cinnamaldehyde. ${ }^{[83]}$

The dual responsive carriers for colorectal cancer applications are used for synergistic purposes rather than the sequential therapeutic protection and sustained release strategy that is typically observed in IBD applications. However, these $\mathrm{pH}$-responsive systems that degrade under more mild acidic conditions can still be utilized as an additional protective layer for delivery in the GIT. For example, within the $\mathrm{GI}$ tract the $\mathrm{pH}$ drops to 5.7 in the caecum and gradually increases to $\mathrm{pH} 6.7$ in the rectum. ${ }^{[84]}$ Moreover, there are different forms of IBD that possess varying levels of inflammation within the colon. IBD associated with primary sclerosing cholangitis, for example, has the highest histologically graded inflammation in the proximal colon. ${ }^{[85]}$ Moreover, distal ulcerative colitis demonstrated higher inflammation in the distal colon. ${ }^{[86]}$ Thus, incorporating an additional $\mathrm{pH}$-sensitive layer can further localize the therapeutic release to the specific site of interest.

\subsection{3 | External stimuli and future considerations for multi-responsive drug delivery systems for IBD therapy}

Finally, external stimuli also serve as a promising strategy for increasing GIT targeting in a remote, patient-specific manner. Internal stimuli can often lead to off-targeting drug release due to patient-specific differences in the GI tract environment. Externally triggered systems are promising tools in modern precision medicine, because the physician has spatiotemporal control over the therapeutic delivery. Computed tomography (CT) contrast agents were loaded into thermoresponsive capsules and released via magnetic hyperthermia to image the drug payload location in the gut. ${ }^{[87]}$

Moreover, low-frequency ultrasound is being investigated to improve drug penetration in the colonic mucosa. ${ }^{[88-92]}$ Drug delivery to the colonic mucosa requires that the carrier diffuses through the mucus and enter the mucosal tissue via the epithelial enhanced permeability and retention effect. ${ }^{[91]}$ Ultrasound can permeabilize biological membranes to increase the diffusion of the therapeutic through tissue barriers, with potential applications in GIT delivery. Schoellhammer et al. ${ }^{\text {[92] }}$ demonstrated in an ex vivo experiment that exposure to lowfrequency ultrasound enhanced the delivery of dextran by almost 7-fold in comparison to passive diffusion. Schoellhammer et al. also investigated the optimal permeant characteristics for ultrasoundmediated GI delivery, including the effect of permeant size, charge, and the presence of chemical penetration enhancers. ${ }^{[90]}$

\section{6 | CONCLUSIONS AND FUTURE OUTLOOK}

The biggest challenge in $\mathrm{Gl}$ drug targeting is reaching a maximal local drug exposure at the inflamed regions, while avoiding unspecific drug delivery and systemic exposure. By using nanotechnology for drug delivery, the therapeutic effect may be achieved, while side-effects are potentially reduced. Depending on the different locations of the inflamed area, drug delivery systems must overcome multiple 
physiological barriers in order to maximize drug availability, promisingly addressing the existing medical challenges.

ROS-responsive drug delivery systems, which degrade in the presence of a pathophysiological level of oxidative stress, coated with different stimuli-responsive materials, provide outstanding drug protection capacities from the physiological conditions throughout the Gl tract, enabling a disease-specific targeting approach while maintaining drug integrity until it reaches the inflamed areas, as shown by the examples covered in this review.

Nevertheless, there is still a great gap between the laboratory validation and clinical translation. Currently, no clinical trials have been documented to further testify the function of the ROS-responsive drug delivery systems. Therefore, further studies are needed to demonstrate its potential to treat $\mathrm{GI}$ diseases.

Moreover, while external stimuli such as ultrasounds have not been applied in conjunction with internal stimuli for IBD therapeutic delivery applications, combining the previously discussed internal and external stimuli can potentially increase the therapeutic efficacy at the inflamed site of interest. Moreover, triggering a release via external means at different locations within the GIT or increasing mucosa permeability in conjunction with protecting the therapeutic at acid $\mathrm{pH}$ in the upper GIT would produce more tailored formulations for IBDs with varying severity, locations, and pathogenesis. Because drug delivery vectors need to overcome multiple physiological barriers as they travel from the oral cavity to inflamed intestine, multi-stimuli responsive materials are required to protect the cargo and ensure high drug concentrations reach the inflamed sites. Thus, it is important that we have materials that not only respond to obstacles present in the upper GIT, but also more specifically, to the microenvironment at the site of action through either internal, external or a combination of stimuli responsive carriers.

However, special attention should be paid to the dosage and selection of loaded drug molecules to avoid anti-oxidant-induced stress. Overall, ROS-responsive systems hold great potential to selectively deliver drugs to inflamed areas and effective decrease inflammation throughout the GIT.

\section{ACKNOWLEDGEMENTS}

M. Tiboni acknowledges the Italian Ministry of Education, University and Research for the "Bando Leonardo da Vinci - Azione 2" travel grant. Prof. H. A. Santos acknowledges financial support from the Sigrid Jusélius Foundation, the HiLIFE Research Funds, and the Academy of Finland (decision no. 319330).

\section{ORCID}

Hélder A. Santos (D) https://orcid.org/0000-0001-7850-6309

\section{REFERENCES}

[1] J. F. Pinto, Int. J. Pharm. 2010, 395, 44.

[2] S. Sharma, V. R. Sinha, J. Control. Release 2018, 272, 97.

[3] M. Wei, Y. Gao, X. Li, M. J. Serpe, Polym. Chem. 2017, 8, 127.
[4] M. Faria, M. Björnmalm, K. J. Thurecht, S. J. Kent, R. G. Parton, M. Kavallaris, A. P. R. Johnston, J. J. Gooding, S. R. Corrie, B. J. Boyd, P. Thordarson, A. K. Whittaker, M. M. Stevens, C. A. Prestidge, C. J. H. Porter, W. J. Parak, T. P. Davis, E. J. Crampin, F. Caruso, Nat. Nanotechnol. 2018, 13, 777.

[5] J. R. Turner, Nat. Rev. Immunol. 2009, 9, 799.

[6] M. Coskun, Front. Med. 2014, 1, 1.

[7] R. Okumura, K. Takeda, Exp. \&Amp; Mol. Med. 2017, 49, e338.

[8] F. Araújo, J. das Neves, J. P. Martins, P. L. Granja, H. A. Santos, B. Sarmento, Prog. Mater. Sci. 2017, 89, 306.

[9] M. Wahlgren, M. Axenstrand, Å. Håkansson, A. Marefati, B. Lomstein Pedersen, Pharmaceutics 2019, 11, 95.

[10] C. A. S. Bergström, R. Holm, S. A. Jørgensen, S. B. E. Andersson, P. Artursson, S. Beato, A. Borde, K. Box, M. Brewster, J. Dressman, K.-I. Feng, G. Halbert, E. Kostewicz, M. McAllister, U. Muenster, J. Thinnes, R. Taylor, A. Mullertz, Eur. J. Pharm. Sci. 2014, 57, 173.

[11] S. Edsbäcker, B. Bengtsson, P. Larsson, P. Lundin, Å. Nilsson, J. Ulmius, P. Wollmer, Aliment. Pharmacol. Ther. 2003, 17, 525.

[12] R. B. Sartor, Gastroenterology 2008, 134, 577.

[13] A. Bak, M. Ashford, D. J. Brayden, Adv. Drug Deliv. Rev. 2018, 136-137, 2.

[14] U. Klotz, M. Schwab, Adv. Drug Deliv. Rev. 2005, 57, 267.

[15] S. Hua, E. Marks, J. J. Schneider, S. Keely, Nanomedicine Nanotechnology, Biol. Med. 2015, 11, 1117.

[16] B. Hebden, W. Perkins, Spiller, Aliment. Pharmacol. Ther. 2000, 14, 155.

[17] L. Lih-Brody, S. R. Powell, K. P. Collier, G. M. Reddy, R. Cerchia, E. Kahn, G. S. Weissman, S. Katz, R. A. Floyd, M. J. McKinley, S. E. Fisher, G. E. Mullin, Dig. Dis. Sci. 1996, 41, 2078.

[18] Y.-C. Peng, C.-L. Hsu, C.-F. Tung, W.-K. Chou, L.-R. Huang, D.-Z. Hung, W.-H. Hu, D.-Y. Yang, Hepatogastroenterology. 2008, 55, 770.

[19] H. Ye, Y. Zhou, X. Liu, Y. Chen, S. Duan, R. Zhu, Y. Liu, L. Yin, Biomacromolecules 2019, 20, 2441.

[20] Q. Xu, C. He, C. Xiao, X. Chen, Macromol. Biosci. 2016, 16, 635.

[21] C.-C. Song, F.-S. Du, Z.-C. Li, J. Mater. Chem. B 2014, 2, 3413.

[22] Q. Wang, R. Zhang, M. Lu, G. You, Y. Wang, G. Chen, C. Zhao, Z. Wang, X. Song, Y. Wu, L. Zhao, H. Zhou, Biomacromolecules 2017, 18,1333

[23] B. Khor, A. Gardet, R. J. Xavier, Nature 2011, 474, 307.

[24] D. K. Podolsky, N. Engl. J. Med. 2002, 347, 417.

[25] A. Ballinger, Curr. Gastroenterol. Rep. 2008, 10, 485.

[26] C. Lautenschläger, C. Schmidt, D. Fischer, A. Stallmach, Adv. Drug Deliv. Rev. 2014, 71, 58.

[27] A. Lamprecht, U. Schäfer, C. M. Lehr, Pharm. Res. 2001, 18, 788.

[28] A. Lamprecht, N. Ubrich, H. Yamamoto, U. Schäfer, H. Takeuchi, C.M. Lehr, P. Maincent, Y. Kawashima, J. Control. Release 2001, 71, 297.

[29] J. Shi, A. R. Votruba, O. C. Farokhzad, R. Langer, Nano Lett. 2010, 10, 3223.

[30] W. Tao, Z. He, Asian J. Pharm. Sci. 2018, 13, 101.

[31] J. Liang, B. Liu, Bioeng. Transl. Med. 2016, 1, 239.

[32] M. K. Chourasia, S. K. Jain, Drug Deliv. 2004, 11, 129.

[33] S. Bertoni, Z. Liu, A. Correia, J. P. Martins, A. Rahikkala, F. Fontana, M. Kemell, D. Liu, B. Albertini, N. Passerini, W. Li, H. A. Santos, Adv. Funct. Mater. 2018, $28,1$.

[34] W. Li, Y. Li, Z. Liu, N. Kerdsakundee, M. Zhang, F. Zhang, X. Liu, T. Bauleth-ramos, W. Lian, E. Mäkilä, M. Kemell, Y. Ding, B. Sarmento, R. Wiwattanapatapee, J. Salonen, et al., Biomaterials 2018, 185, 322.

[35] E. Walter, S. Janich, B. J. Roessler, J. M. Hilfinger, G. L. Amidon, J. Pharm. Sci. 1996, 85, 1070.

[36] J. Riikonen, A. Correia, M. Kovalainen, S. Näkki, M. Lehtonen, J. Leppänen, J. Rantanen, W. Xu, F. Araújo, J. Hirvonen, K. Järvinen, H. A. Santos, V.-P. Lehto, Biomaterials 2015, 52, 44.

[37] A. Rahikkala, S. A. P. Pereira, P. Figueiredo, M. L. C. Passos, A. R. T. S. Araújo, M. L. M. F. S. Saraiva, H. A. Santos, Adv. Biosyst. 2018, 2 , 1800020 
[38] N. Kerdsakundee, W. Li, J. P. Martins, Z. Liu, F. Zhang, M. Kemell, A. Correia, Y. Ding, M. Airavaara, J. Hirvonen, R. Wiwattanapatapee, H. A. Santos, Adv. Healthc. Mater. 2017, 6, 1700629.

[39] F. Araújo, N. Shrestha, M.-A. Shahbazi, P. Fonte, E. M. Mäkilä, J. J. Salonen, J. T. Hirvonen, P. L. Granja, H. A. Santos, B. Sarmento, Biomaterials 2014, 35, 9199.

[40] S. Wirtz, C. Neufert, B. Weigmann, M. F. Neurath, Nat. Protoc. 2007, 2, 541.

[41] Q. Zhang, H. Tao, Y. Lin, Y. Hu, H. An, D. Zhang, S. Feng, H. Hu, R. Wang, X. Li, J. Zhang, Biomaterials 2016, 105, 206.

[42] Q. Sun, L. Luan, M. Arif, J. Li, Q. J. Dong, Y. Gao, Z. Chi, C. G. Liu, Carbohydr. Polym. 2018, 189, 352.

[43] P. He, S. S. Davis, L. Illum, Int. J. Pharm. 1998, 166, 75.

[44] D. S. Wilson, G. Dalmasso, D. Merlin, N. Murthy, L. Wang, S. V. Sitaraman, Nat. Mater. 2010, 9, 923.

[45] M. Naeem, J. Cao, M. Choi, W. S. Kim, H. R. Moon, B. L. Lee, M.S. Kim, Y. Jung, J. Yoo, Int. J. Nanomedicine 2015, 10, 4565.

[46] M. Naeem, W. Kim, J. Cao, Y. Jung, J. Yoo, Colloids Surfaces B Biointerfaces 2014, 123, 271.

[47] S. H. Lee, M. K. Gupta, J. B. Bang, H. Bae, H. J. Sung, Adv. Healthc. Mater. 2013, 2, 908.

[48] M. Leirdal, M. Sioud, Biochem. Biophys. Res. Commun. 2002, 295, 744.

[49] D. Palliser, D. Chowdhury, Q.-Y. Wang, S. J. Lee, R. T. Bronson, D. M. Knipe, J. Lieberman, Nature 2006, 439, 89.

[50] N. Murata, Y. Takashima, K. Toyoshima, M. Yamamoto, H. Okada, J. Control. Release 2008, 126, 246.

[51] S. Akhtar, I. F. Benter, J. Clin. Invest. 2007, 117, 3623.

[52] L. Thiele, B. Rothen-Rutishauser, S. Jilek, H. Wunderli-Allenspach, H. P. Merkle, E. Walter, J. Control. Release 2001, 76, 59.

[53] S. Hariharan, V. Bhardwaj, I. Bala, J. Sitterberg, U. Bakowsky, M. N. V. Ravi Kumar, Pharm. Res. 2006, 23, 184.

[54] M. P. Desai, V. Labhasetwar, G. L. Amidon, R. J. Levy, Pharm. Res. 1996, 13, 1838.

[55] H. T. T. Nguyen, D. Merlin, Cell. Mol. Life Sci. 2012, 69, 3015.

[56] A. C. Santos Akkari, A. F. Keppler, D. R. de Araujo, E. V. Ramos Campos, L. F. Fraceto, E. de Paula, G. R. Tófoli, Colloids Surfaces B Biointerfaces 2016, 138(138-147), 138.

[57] J. Hombach, H. Hoyer, A. Bernkop-Schnürch, Eur. J. Pharm. Sci. 2008, 33, 1.

[58] C. De Gracia Lux, S. Joshi-Barr, T. Nguyen, E. Mahmoud, E. Schopf, N. Fomina, A. Almutairi, J. Am. Chem. Soc. 2012, 134, 15758.

[59] S. Zhang, R. Langer, G. Traverso, Nano Today 2017, 16, 82.

[60] J. Kost, R. Langer, Adv. Drug Deliv. Rev. 2001, 46, 125.

[61] Y. Lu, A. A. Aimetti, R. Langer, Z. Gu, Nat. Rev. Mater. 2017, 2(1), 16075 .

[62] S. Zhang, J. Ermann, M. D. Succi, A. Zhou, M. J. Hamilton, B. Cao, J. R. Korzenik, J. N. Glickman, P. K. Vemula, L. H. Glimcher, G. Traverso, R. Langer, J. M. Karp, Sci. Transl. Med 2015, 7, 300ra128.

[63] R. Kinget, W. Kalala, L. Vervoort, G. van den Mooter, J. Drug Target. 1998, 6, 129.

[64] M. Zhang, D. Merlin, Inflamm. Bowel Dis. 2018, 24, 1401.

[65] S. Yadav, S. R. Deka, K. Tiwari, A. K. Sharma, P. Kumar, IEEE Trans. Nanobioscience 2017, 16, 764.

[66] C. Kriegel, H. Attarwala, M. Amiji, Adv. Drug Deliv. Rev. 2013, 65, 891.

[67] S. Jain, M. Amiji, Nanoparticles-in-Microsphere Oral Delivery Systems (NiMOS) for Nucleic Acid Therapy. Mucosal Delivery of Biopharmaceuticals: Biology, Challenges and Strategies, USA Springer, Boston, MA 2014, p. 283.

[68] W. J. Sandborn, S. Travis, L. Moro, R. Jones, T. Gautille, R. Bagin, M. Huang, P. Yeung, E. D. Ballard, Gastroenterology 2012, 143, 1218.

[69] D. R. Friend, Adv. Drug Delive 2005, 57, 247.

[70] Q. Zhu, J. Talton, G. Zhang, T. Cunningham, Z. Wang, I. M. Belyakov, R. J. Mumper, J. A. Berzofsky, Nat Med. 2013, 18, 1291.
[71] N. Vergnolle, Gut 2016, 65, 1215.

[72] M. D. Baugh, M. J. Perry, A. P. Hollander, D. R. Davies, S. S. Cross, A. J. Lobo, C. J. Taylor, G. S. Evans, Gatroenterology 1999, 117, 814.

[73] C. Medina, M. W. Radomski, Journal of Pharm and Exp Ther. 2006, 318,933

[74] S. O. Sullivan, J. F. Gilmer, C. Medina, Mediators of inflammation 2015, 2015, 964131.

[75] B. Siegmund, H. Lehr, G. Fantuzzi, C. A. Dinarello, PNAS 2001, 98, 13249.

[76] J. M. Knipe, L. E. Strong, N. A. Peppas, Biomacromolecules 2016, 17, 788.

[77] M. Clinton, J. Frank, M. Gupta, A. Zhang, N. A. Peppas, J. Control. Release 2016, 221, 18.

[78] K. E. Broaders, S. Grandhe, J. M. J. J. Fréchet, J. Am. Chem. Soc. 2011 , 133, 756.

[79] R. B. Sartor, Aliment. Pharmacol. Ther. 2016, 43, 27.

[80] H. L. Lee, S. C. Hwang, J. W. Nah, J. Kim, B. Cha, D. H. Kang, Y. Jeong, J. Pharm. Sci. 2018, 107, 2702.

[81] X. Cheng, Y. Jin, T. Sun, R. Qi, H. Li, W. Fan, Colloids Surfaces B Biointerfaces 2016, 141, 44.

[82] S. Park, B. Kwon, W. Yang, E. Han, W. Yoo, B. Kwon, D. Lee, J. Control. Release 2014, 196(19-27), 19.

[83] J. Noh, B. Kwon, E. Han, M. Park, W. Yang, W. Cho, W. Yoo, G. Khang, D. Lee, Nat. Commun. 2015, 6, 1.

[84] S. G. Nugent, D. Kumar, D. S. Rampton, D. F. Evans, Gut 2001, 48, 571.

[85] A. B. Vries, M. Janse, H. Blokzijl, R. K. Weersma, A. B. Vries, M. Janse, H. Blokzijl, World J. Gastroenterol. 2015, 21, 1956.

[86] I. E. Koutroubakis, World J. Gastrointest. Pharmacol. Ther. 2010, $1,43$.

[87] J. C. Bear, P. S. Patrick, A. Casson, P. Southern, F. Lin, M. J. Powell, Q. A. Pankhurst, T. Kalber, M. Lythgoe, I. P. Parkin, A. G. Mayes, Nat. Publ. Gr. 2016, 6, 1.

[88] C. M. Schoellhammer, R. Langer, G. Traverso, Tissue Barriers 2016, 4 (1-5), e1150235.

[89] C. M. Schoellhammer, G. Traverso, Expert Opin. Drug Deliv. 2016, 13, 1045.

[90] C. M. Schoellhammer, Y. Chen, C. Cleveland, D. Minahan, T. Bensel, J. Y. Park, S. Saxton, Y. L. Lee, L. Booth, R. Langer, G. Traverso, J. Control. Release 2017, 268, 113.

[91] Q. Chen, B. Xiao, D. Merlin, G. State, V. Affairs, Transl Cancer Res 2017, 6, 10.

[92] C. M. Schoellhammer, G. Y. Lauwers, J. A. Goettel, M. A. Oberli, C. Cleveland, J. Y. Park, D. Minahan, Y. Chen, D. G. Anderson, A. Jaklenec, S. B. Snapper, R. Langer, G. Traverso, Gastroenterology 2017, 152, 1151.

\section{AUTHOR BIOGRAPHIES}

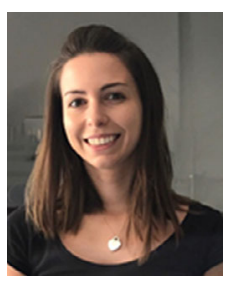

Serena Bertoni received PhD in Biotechnological and Pharmaceutical Sciences in March 2019 from the University of Bologna (Italy). Currently, she is a post-doctoral fellow in the pharmaceutical technology research group of Nadia Passerini and Beatrice Albertini at the University of Bologna. Her research activity is focused on the development of oral drug delivery systems, mainly polymeric/lipidic microparticles for the delivery of biological drugs (protein, peptides, enzymes) and formulation of drugs with limited bioavailability. 


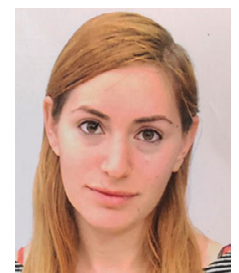

Ariella Machness is a PhD student in Materials Science and Engineering at the University of California, Los Angeles under the supervision of Prof Mark Goorsky. She is currently a visiting researcher at Prof. Helder Santos' group as a joint National Science Foundation- Academy of Finland fellow. Her research interests include magnetic nanoparticles and their application in stimuli-responsive drug delivery devices.

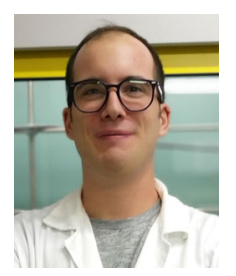

Mattia Tiboni (M.Sc. Pharm.) obtained his Master's Degree in Medicinal Chemistry and Pharmaceutical Technology at the University of Urbino Carlo Bo (Italy). He is currently a PhD student in Pharmaceutical Sciences at the same University under the supervision of Prof. Luca Casettari. He has been visiting PhD student in Professor Santos' Lab focusing on anticancer nanomedicine. His main research interest is the development of innovative biomimetic drug delivery systems.

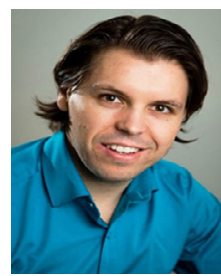

HélDer A. Santos obtained his Doctor of Science in Technology (Chemical Engineering) in 2007 from the Helsinki University of Technology (Finland). Currently, he is an Associate Professor in the Faculty of Pharmacy, University of Helsinki (Finland) where he serves as Head of the Division of Pharmaceutical Chemistry and Technology, Head of the Preclinical Drug Formulation and Analysis Group, and Head of the Nanomedicines and Biomedical Engineering research group. His scientific expertise lies in the development of nanoparticles/nanomedicines for biomedical applications, particularly porous silicon and polymeric-based nanomaterials for simultaneous controlled drug delivery, diagnostic, and therapy for cancer, diabetes, and cardiovascular diseases.

How to cite this article: Bertoni S, Machness A, Tiboni M, Bártolo R, Santos HA. Reactive oxygen species responsive nanoplatforms as smart drug delivery systems for gastrointestinal tract targeting. Biopolymers. 2019;e23336. https://doi.org/10.1002/bip.23336 